\title{
Reliable Redundant Control for Nonlinear uncertain Systems
}

\author{
Zhang Yingwei Wang Fuli \\ (Institute of Control System \& Intelligent Instrument, Northeastern University. Shenyang 110004. China) \\ E-mail address:zhang_yingwei2001@yahoo.com.cn
}

\begin{abstract}
This paper is concerned on the reliable control problem of the nonlinear systems that experience the single fault. By using strictly redundant control elements, the methods to design reliable controller are presented. The resulting closed-loop nonlinear systems are reliable in the sense that they provide guaranteed local stability and $\mathrm{H}_{\infty}$ performance not only all sensors and actuators are operational, but also when any one, but only one, sensor or actuator experiences fault.
\end{abstract}

Key words: reliable control; $\mathrm{H}_{\infty}$ control; nonlinear system; robust control

\section{Introduction}

In recent years, there has some important achievement in the design problems of reliable control systems. Vidyasagar and Viswanadham discuss the reliable stabilization of a plant by two controllers summed together by means of factorization methods and given any stabilizing controller for a plant, a procedure of designing a second stabilizing controller such that the sum of the two controllers also stabilizes the plant. Tan et al. investigated the reliable stabilization problem of multicontroller systems composed of one plant and two controllers. Gundes and Kabuli investigate the reliable stabilization problem for twochannel decentralized control systems and present reliable decentralized controller design methods for strongly stabilizable plants. Another reliability goal is to provide guaranteed system performance. Veillette et al. present a new methodology for the design of reliable centralized and decentralized control systems by using the algebraic Riccati equation approach, where the resulting designs provide guaranteed closed-loop stability and $\mathrm{H}_{\infty}$ performance not only when all control component failures. In particular, Var der Schaft has shown that the solution of the $\mathrm{H}_{\infty}$ control problem via state feedback can be determined from the solution of a Hamilton-Jacobi equation (or inequality). Siljak investigated reliability of control structures using more than one controller for a given plant, which is a natural way to introduce redundancy into a control scheme for enhancing reliability. Yang discuss reliable nonlinear control system design by using strictly redundant control elements.

This paper will discuss the reliable redundant controller design problem for nonlinear uncertain systems using Hamilton-Jacobi inequality. The closed-loop systems are stable when all sensors and actuators are functioning but also when only one sensors or actuator experiences a failure or outage.

\section{Problem statement}

Consider a nonlinear uncertain systems $\Sigma$ as follows:

$$
\begin{aligned}
\Sigma: \quad \dot{x} & =f(x)+\Delta f(x)+g_{1}(x) w_{0}+\sum_{j=1}^{m} g_{2 j}(x) u_{j}, \\
y_{i} & =h_{2 i}(x)+w_{i}, \quad i=1,2, \cdots, q \\
z & =\left[\begin{array}{llll}
h_{1}^{T}(x) & u_{1} & \cdots & u_{m}
\end{array}\right] \in R^{s}, \quad s=p+m
\end{aligned}
$$

where $x$ is the state vector defined in a bounded neighbourhood $X$ of the origin in $\mathrm{R}^{\mathrm{n}}, w_{0} \in \mathrm{R}^{q_{0}}$ is the disturbance, $u=\left[u_{1}, u_{2}, \cdots, u_{m}\right]^{T} \in \mathbf{R}^{m}$ is the control input, $w=\left[w_{1}, w_{2}, \cdots, w_{q}\right] \in \mathrm{R}^{q}$ is the measurement noise, $y=\left[y_{1}, y_{2}, \cdots, y_{q}\right]^{T} \in \mathbb{R}^{q}$ is the measured output; $z \in R^{s}$ is the output to be regulated.

The function $f(x), g_{1}(x), h_{1}(x), g_{2 j}(x)(j=1, \cdots, m)$ and $h_{2 i}(x) \quad(i=1, \cdots, q)$ are all known smooth mappings of appropriate dimensions and are defined in the neighbourhood $X$ of $0 \in \mathbb{R}^{n}$ with $f(0)=0, h_{1}(0)=0$ and $h_{2 i}(0)=0(i=1, \cdots, q)$. We denote:

$$
\begin{aligned}
& w_{r}=\left[\begin{array}{ll}
w_{0}^{T} & w^{T}
\end{array}\right] \equiv \mathrm{R}^{r} \quad r=q_{0}+q \\
& g_{2}(x)=\left[\begin{array}{llll}
g_{21}(x) & g_{22}(x) & \cdots & g_{2 m}(x)
\end{array}\right] \\
& h_{2}(x)=\left[\begin{array}{lllll}
h_{21}(x) & h_{22}(x) & \cdots & h_{2 q}(x)
\end{array}\right]^{r}
\end{aligned}
$$

suppose smooth uncertain mapping $\Delta f(x)$ are normbounded

$\Delta f(x) \in \Omega_{f} \triangleq\left\{f_{01}(x) \alpha_{f}(x): \alpha_{f}^{T}(x) \alpha_{f}(x) \leq f_{02}^{T}(x) f_{02}(x), x \in \mathrm{X}\right\}$,

where $f_{01}(x)$ are known smooth matrix mappings, with $f_{02}(0)=0$. Then, the problem considered in this paper is as follows

Single fault reliable redundant control (SFRRC) problem: Given the system $\Sigma$ of (1)-(3) and a positive constant $\gamma$, design a controller of the following form:

$$
\begin{aligned}
& \dot{\xi}=a(\xi)+b(\xi) y, \quad \xi \in R^{\nu} \\
& u(\xi)=c(\xi)
\end{aligned}
$$

such that the resulting closed-loop system has a local $L_{2}$ gain less than or equal to $\gamma$.

Definition 1: Let $F^{\prime}(x)=\left[F_{1}(x) F_{2}(x) \cdots F_{m}(x)\right]$ be a smooth matrix-valued function. Then the matrix $F(x)$ is called strictly redundani if the $m$ column vectors $F_{1}(x), F_{2}(x), \cdots, F_{m}(x)$ are functionally dependent, but any $m-I$ of these $m$ column vector are functionally independent. 
Definition 2: Let $E(x)$ be a smooth matrix-valued function with $E(0)=0$. Then the function $E(x)$ is called strictly redundant if there exists a smooth matrix-valued function $E^{0}(x)$ such that $E(x)=E^{0}(x) x$ and such that $E^{0}(x)^{T}$ is strictly redundant.

The following lemma presents basic property for strictly redundant function.

Lemma 1:(i) Suppose that the matrix-valued function $F(x)=\left[F_{1}(x) F_{2}(x) \cdots F_{m}(x)\right]$ is strictly redundant. Then there exists a positive function $\delta_{F}(x)<1$ for $x \in X$, such that

$$
F_{i}(x) F_{i}^{T}(x) \leq \delta_{F}(x) F(x) F^{T}(x) \quad, \quad i=1, \cdots, m, \quad x \in X
$$

(ii) Suppose that the matrix-valued function $E(x)=\left[E_{1}(x) E_{2}(x) \cdots E_{m}(x)\right]: X \rightarrow R^{m}$ is called strictly redundant if there exists a positive smooth scalar function $\delta_{E}(x)<1$ with $x \in X$, such that

$E_{i}^{2}(x) \leq \delta_{E}(x) E^{T}(x) E(x), \quad i=1, \cdots, m, \quad x \in X$

\section{Preliminaries} following:

Consider the system $\Sigma$ of (1)-(3) and suppose the

Assumption 1 The function $g_{2}(x)$ and $h_{2}(x)$ are strictly redundant, we known from lemma 1 ,exits positive smooth function $0<\delta_{g_{2}}(x)<1$ and $0<\delta_{h_{2}}(x)<1$ such that following inequalities hold:

$$
\begin{aligned}
& g_{2 i}(x) g_{2 i}^{T}(x) \leq \delta_{g_{2}}(x) g_{2}(x) g_{2}^{T}(x), i=1, \cdots, m, \quad \forall x \in X \\
& h_{2 i}^{2}(x) \leq \delta_{h_{2}}(x) h_{2}^{T}(x) h_{2}(x), i=1, \cdots, q, \forall x \in X \\
& \text { when } \omega_{s} \subset \Omega_{s} \\
& \text { Let } U(x), V(x) \text { be } C^{\prime} \text { function, denote } \\
& H_{s}\left(V_{x}, x, \delta_{g_{2}}, \delta_{h_{2}}\right)=V_{x} f(x)+\delta_{h_{2}}(x) \gamma^{2} h_{2}^{T}(x) h_{2}(x)+ \\
& \frac{1}{4} V_{x}\left(\frac{1}{\gamma^{2}} g_{10}(x) g_{10}^{T}(x)-\left[1-\delta_{g_{2}}(x)\right] g_{2}(x) g_{2}^{T}(x)\right) V_{x}^{T} \\
& H_{0}\left(V_{x}, x, \delta_{g_{2}}, \delta_{h_{2}}\right)=U_{x} f(x)-\left[1-\delta_{h_{2}}(x)\right] \gamma^{2} h_{2}^{T}(x) h_{2}(x)+ \\
& \frac{1}{4 \gamma^{2}} U_{x} g_{10}(x) g_{10}^{T}(x) U_{x}^{T}+\frac{1}{4} \delta_{g_{2}}(x) U_{x} g_{2}(x) g_{2}^{T}(x) U_{x}^{T}
\end{aligned}
$$

where $U_{\mathrm{x}}$ and $V_{\mathrm{x}}$ are the Jacobian matrices of $U$ and $V$ respectively.

Assumption 2 There are some smooth function $\varphi(x) \geq 0$ with $\varphi(0)=0$ such that

(a) the Hamilton-Jacobi equation

$H_{s}\left(V_{x}, x, \delta_{g_{2}}, \delta_{h_{2}}\right)+\varphi(x)=0$

has a $C^{3}$ positive definite solution $V(x)$ with $V(0)=0$;

(b) the Hamilton-Jacobi inequality

$H_{0}\left(U_{x}, x, \delta_{g_{2}}, \delta_{h_{2}}\right)+\varphi(x) \leq 0$ has a $C^{3}$ positive definite solution $U(x)$ with $U(0)=0$ and $H_{0}\left(U_{x}, x, \delta_{g_{2}}, \delta_{h_{2}}\right)+\varphi(x)$ has non-singular Hessian matrix at zero;

(c) $U(x)-V(x)$ is positive definite and

$$
\left[U_{x}(x)-V_{x}(x)\right] L(x)=2 \gamma^{2} h_{2}^{T}(x)
$$

has a solution $L(x)$.

\section{Main results}

Theorem 1: Consider the system $\Sigma$ of (1)-(3), under the assumption 1 and 2 , then the controller (8) and (9) with the following

$$
\begin{aligned}
& a(\xi)=f(\xi)+\frac{1}{2 \gamma^{2}} g_{10}(\xi) g_{10}^{T}(\xi) V_{x}^{T}(\xi)- \\
& \frac{1}{2}\left[1-\delta_{g_{2}}(\xi)\right] g_{2}(\xi) g_{2}^{T}(\xi) V_{x}^{T}(\xi)-L(\xi) h_{2}(\xi) \\
& b(\xi)=L(\xi) \\
& c(\xi)=-\frac{1}{2} g_{2}^{T}(\xi) V_{x}^{T}(\xi)
\end{aligned}
$$

solve the SFRRC problem with local stability, where

$$
\begin{aligned}
& g_{10}(x)=\left[\begin{array}{lll}
g_{1}(x) & \gamma \sqrt{\lambda_{1}(x)} f_{01}(x) \quad \gamma \sqrt{\lambda_{2}(x)} \lambda h_{01}(x)
\end{array}\right. \\
& \left.\gamma_{2 \Omega,}(x)\right] \text {. }
\end{aligned}
$$

\section{Conclusion}

This paper discussed single fault reliable control with redundant element for uncertain nonlinear systems. We solve uncertainty using by introduce of extended systems.

\section{Referances}

[1] M. Vidyasagar and N. Viswanadham (1985) "Reliable stabilization using a multicontroller configuration," Automatica, vol. 21, no. 5, pp. 599602.

[2] X. L. Tan, Dragoslav D. Siljak, and Masao Ikeda (1991). "Reliable stabilization via factorization methods", IEEE Trans. Automat. Contr., vol. 37, pp. 1786-1791.

[3] A. N. Gundes and M. G. Kabuli, "Reliable decentralized control," in Proc. American Control Conf., Baltimore, MD, pp. 3359-3363.

[4] R. J. Veillette (1992). J. V. Medanic, and W. R. Perkings, "Design of reliable control systems," IEEE Trans. Automat. Contr., vol. 37, pp. 290-304.

[5] Van Der Schaft (1992). $\mathrm{L}_{2}$-gain analysis of nonlinear systems and nonlinear control. IEEE Transactions on Automatic Control, 34,770-784.

[6] D. D. Siljak (1980), "Reliable contril using multiple control systems," Int. J. Contr., vol. 31 , no. 2, pp. 303-329.

[7] G. H. Yang, J. L. Wang and C. B.Soh(1998a). Reliable nonlinear control system design by using strictly redundant control elements. International Journal of Control, 69(2), 315-328. 